\title{
SOME INFINITELY GENERATED NON-PROJECTIVE MODULES OVER PATH ALGEBRAS AND THEIR EXTENSIONS UNDER MARTIN'S AXIOM
}

\author{
AYAKO ITABA, DIEGO A. MEJÍA, AND TERUYUKI YORIOKA
}

\begin{abstract}
In this paper it is proved that, when $Q$ is a quiver that admits some closure, for any algebraically closed field $K$ and any finite dimensional $K$-linear representation $\mathcal{X}$ of $Q$, if $\operatorname{Ext}_{K Q}^{1}(\mathcal{X}, K Q)=0$ then $\mathcal{X}$ is projective (Theorem 1.10. In contrast, we show that if $Q$ is a specific quiver of the type above, then there is an infinitely generated non-projective $K Q$-module $M_{\omega_{1}}$ such that, when $K$ is a countable field, $\mathrm{MA}_{\aleph_{1}}$ (Martin's Axiom for $\aleph_{1}$ many dense sets, which is a combinatorial axiom in set theory) implies that $\operatorname{Ext}_{K Q}^{1}\left(M_{\omega_{1}}, K Q\right)=0$ (TheOREM 2.11).
\end{abstract}

\section{INTRODUCTION}

Bound quiver algebras of finite connected quivers strongly influence research on representation theory of Artin algebras. Gabriel found a correspondence between finite dimensional algebras and linear representations of bound quivers ([13], 3], II]), so it follows that studying modules of finite dimensional algebras is reduced to studying modules of bound quiver algebras. In this paper, we concentrate on the study of path algebras, which is one type of bound quiver algebras.

Nakayama Conjecture, Tachikawa Conjecture, and Auslander-Reiten Conjecture are some major research projects in ring theory that present sufficient conditions for projective modules. Related to this, it has been known the following result for Artin algebras: $(*)$ For any finite dimensional algebr $* 11$ closed field of finite global dimension and any finitely generated $\Lambda$-module $M$, if $\operatorname{Ext}_{\bar{\Lambda}}^{\geq 1}(M, \Lambda)=0$, then $M$ is projective (TheOREM 1.2). A typical example of finite dimensional algebras is a path algebra of a finite acyclic quiver over an algebraically closed field. Since any path algebra of a quiver over an algebraically closed field is hereditary (even when the quiver is not finite, see e.g. [14, §8.2]), that is, its global dimension is not larger than 1, the following assertion also holds: For any algebraically closed field $K$, any finite acyclic quiver $Q$ and any finitely generated $K Q$-module $M$, if $\operatorname{Ext}_{K Q}^{1}(M, K Q)=0$, then $M$ is projective. In Theorem 1.10. it is shown that the above assertion is also true for finite dimensional $K$-linear representations of some infinite quivers, one of which is the following quiver of $A_{\infty}$ type, denoted by $A_{\infty}^{\leftarrow}$ :

$$
0 \longleftarrow 1 \longleftarrow 2 \longleftarrow \cdots \longleftarrow n \longleftarrow n+1 \longleftarrow \cdots .
$$

2010 Mathematics Subject Classification. 16G10, 16G20, 03E35, 03E50.

Key words and phrases. path algebras, quiver representations, non-projective modules, Martin's Axiom .

${ }^{* 1}$ Any finite dimensional algebra is Artin. 
Let $A_{\infty}$ be the opposite quiver of $A_{\infty}^{\leftarrow}$ : the set of the vertices of $A_{\infty}$ is the same as $A_{\infty}^{\leftarrow}$, but the arrows are reversed, that is, each arrow in $A_{\infty}$ is of the form $n \rightarrow n+1$. The category $\operatorname{Mod} K A_{\infty}$ of $K A_{\infty} \rightarrow$-modules is known to be somewhat simple, actually is pure semisimple, that is, every $K A_{\infty}$-module is a direct sum of finitely presented $K A_{\infty}$-modules [5, $\S 2$ ]. In [7, Theorem 3.1], a characterization

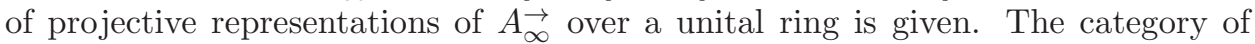
representations of $A_{\infty}^{\leftarrow}$ also has been studied, for example, a characterization of projective representations of $A_{\infty}^{\leftarrow}$ over a field is presented in [4, p102 ExAmPLE], and this is extended to such representations over a unital ring in $[8, \S 3]$.

In this paper, we consider some specific quivers $Q$, as specified in THEOREM 2.11 one of which is the quiver $A_{\infty}^{\leftarrow}$ to construct an infinitely generated non-projective $K Q$-module, which is denoted by $M_{\omega_{1}}$. To analyze such a $K Q$-module $M_{\omega_{1}}, \mathrm{MA}_{\aleph_{1}}$ (Martin's Axiom for $\aleph_{1}$ many dense sets) is used. $\mathrm{MA}_{\aleph_{1}}$ is a combinatorial axiom of set theory that cannot neither be proved nor refuted from Zermelo-Fraenkel axiomatic set theory ZFC with the axiom of choice [18, 21. $M_{\aleph_{1}}$ is applied in many areas of mathematics to show that some mathematical statements cannot be refuted from ZFC (see e.g. [12]). One of such examples is Shelah's solution of Whitehead Problem 20. Our main result states that if $K$ is a countable field and $\mathrm{MA}_{\aleph_{1}}$ holds, then $\operatorname{Ext}_{K Q}^{1}\left(M_{\omega_{1}}, K Q\right)=0$ (Theorems 2.4, 2.9 and TheOREM 2.11). Therefore, under $\mathrm{MA}_{\aleph_{1}}$ and the assumption that $K$ is a countable field, the above assertion $(*)$ fails for quivers $Q$ as in THEOREM 2.11 and infinitely generated $K Q$-modules. Trlifaj's construction is used to build such infinitely generated $K Q$ modules, which will be presented in 1.4 .

This paper is intended to be fairly self contained, but we will assume some basic knowledge about ordinals (see e.g. [11, II.1, II.4] and [17, I.7, III.6]). \$1 provides necessary knowledge, which includes some facts on path algebras and set theory. $\$ 2$ provides the proof of the main result of this paper.

\section{Preliminaries}

Throughout this paper, a ring $R$ means a ring with enough idempotents (hence $R$ may not be unital), and an $R$-module means right $R$-module. For a ring $R$, $\operatorname{Mod} R$ denotes the category of the $R$-modules, and $\bmod R$ denotes the category of the finitely generated $R$-modules. For an $R$-module $M$ and a subset $X$ of $M,\langle X\rangle_{R}$ denotes the $R$-submodule of the module $M$ generated by $X$. For an $R$-module $M$ and $R$-submodules $N_{i}, i \in I$, of $M, \sum_{i \in I} N_{i}$ denotes the $R$-submodule that is the $R$-linear span of the set $\bigcup_{i \in I} N_{i}$.

We follow the notation of outer direct sums in [11, I.2.]. For a family $\left\{M_{i}: i \in I\right\}$ of modules, the product module $\prod_{i \in I} M_{i}$ is the module whose underling set is the set of functions $f$ with domain $I$ such that for each $i \in I, f(i)$ belongs to the set $M_{i}$, and the operations are defined coordinate-wise. For a member $f$ of the product $\prod_{i \in I} M_{i}$, the support $\operatorname{supp}(f)$ of $f$ is defined by the set

$$
\left\{i \in I: f(i) \neq 0_{M_{i}}\right\} \text {. }
$$


The outer direct sum $\bigoplus_{i \in I} M_{i}$ of a family $\left\{M_{i}: i \in I\right\}$ of modules is the submodule of the product module $\prod_{i \in I} M_{i}$ which consists of the members of the set $\prod_{i \in I} M_{i}$ whose supports are finite.

We adopt ordinals as the von Neumann ordinals, that is, an ordinal $\alpha$ means the set of ordinals less than $\alpha$. So for ordinals $\alpha$ and $\beta, \alpha$ is less than $\beta$ iff $\alpha \in \beta$. $\omega$ is the set of all finite ordinals (non-negative integers), $\omega_{1}$ is the least uncountable ordinal (which is a cardinal). Lim denotes the class of all limit ordinals.

The following is a well-known equivalence about projectivity.

Theorem 1.1 (E.g. 1, Propositions 17.1., 17.2]). For a ring $R$ (with enough idempotents) and an $R$-module $P$, the following statements are equivalent.

(1) For every $R$-epimorphism $f$ from an $R$-module $M$ onto an $R$-module $N$ and $R$-homomorphism $g$ from $P$ into $N$, there exists an $R$-homomorphism $h$ from $P$ into $M$ such that $g=f \circ h$.

(2) Every $R$-epimorphism from an $R$-module onto $P$ splits, that is, it is right invertible.

(3) The functor $\operatorname{Hom}_{R}(P,-)$ within the category $\operatorname{Mod} R$ is exact, that is, for every $R$-module $M, \operatorname{Ext}_{R}^{1}(P, M)=0$.

(4) $P$ is isomorphic to a direct summand of a free $R$-module.

1.1. Path algebras and quiver representations. This subsection is devoted to the basics of representation theory of rings. The readers can skip this subsection if they are familiar with path algebras and quiver representations. Quivers, path algebras, and linear representations of quivers are some basic concepts of representation theory of Artin algebras. Our notation and terminology are fairly standard, see e.g. 2, 3]. In the next paragraphs, we refer to [3, Chapters II-III] for definitions, notation, and terminology.

A quiver denotes a directed graph. Any quiver $Q$ consists of a pair of a set $Q_{0}$ of vertices and a set $Q_{1}$ of arrows. Each arrow $a$ is equipped with its source $s(a)$ and its target $t(a)$. A quiver $Q=\left(Q_{0}, Q_{1}\right)$ is called finite if both $Q_{0}$ and $Q_{1}$ are finite sets. A path of the quiver $Q$ is a finite sequence $a_{0} a_{1} \cdots a_{n}$ of arrows of the quiver $Q$ such that, for each $i$ with $0 \leq i<n$, the target of the arrow $a_{i}$ coincides with the source of the arrow $a_{i+1}$. The path $a_{0} a_{1} \cdots a_{n}$ has length $n+1$. For each vertex $v$ of the quiver $Q$, we agree to associate with it a path of length 0 , called the trivial path or the stationary path at the vertex $v$, which is denoted by $e_{v}$. A cycle is a non-trivial path whose source and target coincide. A quiver is called acyclic if there are no cycles in the quiver. For a quiver $Q, \bar{Q}$ denotes the underlying graph of $Q$ that is obtained from $Q$ by forgetting the orientation of the arrows, and a quiver $Q$ is called connected if the graph $\bar{Q}$ is a connected graph. For a field $K$ and a quiver $Q$, the path algebra $K Q$ of the quiver $Q$ over the field $K$ is the $K$-algebra whose underlying set is the $K$-vector space whose basis is the set of all the paths of the quiver $Q$ (which includes all the stationary paths) such that the product of two paths $a_{0} a_{1} \cdots a_{m-1}$ and $b_{0} b_{1} \cdots b_{n-1}$ is defined as follows:

$$
a_{0} a_{1} \cdots a_{m-1} \cdot b_{0} b_{1} \cdots b_{n-1}= \begin{cases}a_{0} a_{1} \cdots a_{m-1} b_{0} b_{1} \cdots b_{n-1} & \text { if } t\left(a_{m-1}\right)=s\left(b_{0}\right) \\ 0_{K Q} & \text { otherwise. }\end{cases}
$$


The product of basic elements is extended to arbitrary elements of $K Q$ by distributivity. We note that, for any field $K$ and a quiver $Q$ with $Q_{0}$ finite, $K Q$ also has an identity, which is of the form $\sum_{v \in Q_{0}} e_{v}$. However, for any quiver $Q$ with infinitely many vertices, $K Q$ does not have an identity. We recall that any path algebra $K Q$ of a quiver $Q$ over an algebraically closed field $K$ is hereditary even when a quiver $Q$ is not finite (see e.g. [14, $\$ 8.2]$ ), that is, its global dimension is not larger than 1 .

For a quiver $Q=\left(Q_{0}, Q_{1}\right)$ and a field $K$, a $K$-linear representation of the quiver $Q$ is a system $\mathcal{X}=\left\langle\mathcal{X}_{v}, \mathcal{X}_{a}: v \in Q_{0}, a \in Q_{1}\right\rangle$ such that, for each vertex $v \in Q_{0}$, $\mathcal{X}_{v}$ is a $K$-vector space and, for each arrow $a \in Q_{1}, \mathcal{X}_{a}$ is a $K$-linear map from the $K$-vector space $\mathcal{X}_{s(a)}$ into the $K$-vector space $\mathcal{X}_{t(a)}$. A $K$-linear representation is called finite dimensional if each $\mathcal{X}_{v}, v \in Q_{0}$, is a finite dimensional $K$-vector space. For two $K$-linear representations $\mathcal{X}$ and $\mathcal{Y}$, a morphism from $\mathcal{X}$ into $\mathcal{Y}$ is a tuple $\varphi=\left\langle\varphi_{v}: v \in Q_{0}\right\rangle$ such that, for each $v \in Q_{0}, \varphi_{v}$ is a $K$-linear map from the $K$-vector space $\mathcal{X}_{v}$ into the $K$-vector space $\mathcal{Y}_{v}$ and, for each arrow $a \in Q_{1}$, the following diagram commutes:

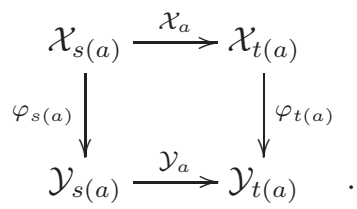

$\operatorname{Rep}_{K} Q$ denotes the category of the $K$-linear representations of a quiver $Q$ over a field $K$, and $\operatorname{rep}_{K} Q$ denotes the category of the finite dimensional $K$-linear representations $\mathcal{X}$ of $Q$ over $K$. In [3], these are defined for finite quivers, however, we adopt them for all quivers.

There is a correspondence between $K Q$-modules and $K$-linear representation of $Q$ (see e.g. [3, Theorem III.1.6]). For a $K Q$-module $M$, define the $K$-linear representation $F(M)$ of $Q$ such that, for each $v \in Q_{0}, F(M)_{v}:=M e_{v}=$ $\left\{m e_{v}: m \in M\right\}$, and, for each $a \in Q_{1}, F(M)_{a}$ is the $K$-homomorphism from $F(M)_{s(a)}$ into $F(M)_{t(a)}$ such that, for each $x \in F(M)_{s(a)}, F(M)_{a}(x)=x a$. For a $K$-linear representation $\mathcal{X}$ of $Q$, define the $K Q$-module $G(\mathcal{X})$ whose underlying set is the direct sum $\bigoplus_{v \in Q_{0}} \mathcal{X}_{v}$ such that, for each element $m=\sum_{v \in Q_{0}} x_{v}$ of $\bigoplus_{v \in Q_{0}} \mathcal{X}_{v}$ (in this notation, for all but finitely many $v \in Q_{0}, x_{v}$ is the zero of $\left.\mathcal{X}_{v}\right), w \in Q_{0}$ and $a \in Q_{1}$, $m e_{w}:=x_{w}$ and $m a:=\mathcal{X}_{a}\left(m e_{s(a)}\right)$, and the product by any arbitrary element of $K Q$ is extended by distributivity. We notice that, for every $K$-linear representation $\mathcal{X}$ of $Q, F(G(\mathcal{X}))=\mathcal{X}$, and, for every $K Q$-module $M$, if $M=\sum_{m \in M} m K Q$ then $G(F(M))=M$. Therefore, if $Q$ is a finite connected quiver, then the category $\operatorname{Mod} K Q$ is equivalent to the category $\operatorname{Rep}_{K} Q$ by the functors $F$ and $G[3$, Theorem III.1.6] and, for any finite acyclic quiver $Q, \bmod K Q$ is equivalent to $\operatorname{rep}_{K} Q[3$, Theorem III.1.7].

1.2. Path algebras of infinite quivers. Throuout this subsection, $F$ denotes the canonical functor from $\operatorname{Mod} K Q$ to $\operatorname{Rep}_{K} Q$, and $G$ denotes the canonical functor from $\operatorname{Rep}_{K} Q$ to $\operatorname{Mod} K Q$, for a field $K$ and a quiver $Q$, as in the last paragraph of the previous subsection. The following theorem gives a sufficient condition for finitely 
generated projective modules over a finite dimensional algebra. For example, the following is mentioned without proof in the proof of [19, Theorem 4.7].

Theorem 1.2 (Folklore). Suppose that $\Lambda$ is a finite dimensional algebra over an algebraically closed field $K$ with finite global dimension. Then for any finitely generated $\Lambda$-module $M$, if $\operatorname{Ext}_{\bar{\Lambda}}^{\geq 1}(M, \Lambda)=0$ then $M$ is projective.

Proof. Suppose that $M$ is a finitely generated $\Lambda$-module and $\operatorname{Ext}_{\Lambda}^{\geq 1}(M, \Lambda)=0$. The point of the proof is to show that, for any finitely generated projective $\Lambda$-module $P, \operatorname{Ext}_{\Lambda}^{\geq 1}(M, P)=0$. To see this, let $P^{\prime}$ be a complementary direct summand of $P$ such that $P \oplus P^{\prime}$ is isomorphic to a direct sum $\Lambda^{n}$ of finitely many copies of $\Lambda$. Then for each integer $k \geq 1$,

$$
\operatorname{Ext}_{\Lambda}^{k}\left(M, \Lambda^{n}\right)=\left(\operatorname{Ext}_{\Lambda}^{k}(M, \Lambda)\right)^{n}=0
$$

and

$$
\operatorname{Ext}_{\Lambda}^{k}\left(M, \Lambda^{n}\right)=\operatorname{Ext}_{\Lambda}^{k}(M, P) \oplus \operatorname{Ext}_{\Lambda}^{k}\left(M, P^{\prime}\right) .
$$

Therefore, $\operatorname{Ext}_{\Lambda}^{k}(M, P)=0$. Hence $\operatorname{Ext}_{\Lambda}^{\geq 1}(M, P)=0$.

Let $d$ be the projective dimension $\operatorname{pd} M$ of $M$. Since $\Lambda$ has finite global dimension, $0 \leq d<\infty$. Assume, towards a contradiction, that $d \geq 1$. Let the sequence

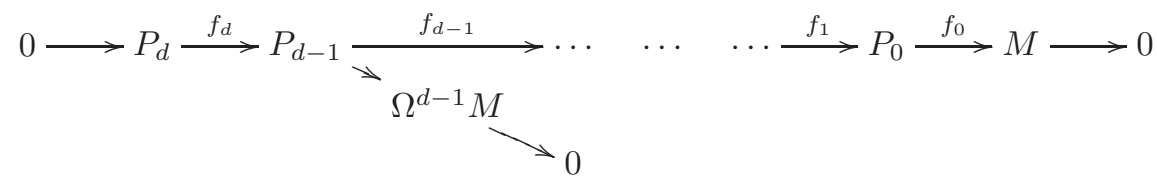

be a projective resolution of $M$ of length $d$ such that each $P_{i}$ is finitely generated, where $\Omega^{d-1} M$ is the $(d-1)$-th syzygy of $M$. Since $\operatorname{pd} M=d$, the projective dimension of the $\Lambda$-module $\Omega^{d-1} M$ is exactly 1 , in particular, $\Omega^{d-1} M$ is not projective. Applying $\operatorname{Hom}_{\Lambda}\left(-, P_{d}\right)$ to the short exact sequence $0 \rightarrow P_{d} \rightarrow P_{d-1} \rightarrow \Omega^{d-1} M \rightarrow$ 0 , we obtain the following exact sequence

$$
\begin{aligned}
0 \rightarrow \operatorname{Hom}_{\Lambda}\left(\Omega^{d-1} M, P_{d}\right) \rightarrow & \\
& \operatorname{Hom}_{\Lambda}\left(P_{d-1}, P_{d}\right) \stackrel{\operatorname{Hom}_{\Lambda}\left(f_{d}, P_{d}\right)}{\longrightarrow} \operatorname{Hom}_{\Lambda}\left(P_{d}, P_{d}\right) \rightarrow \operatorname{Ext}_{\Lambda}^{1}\left(\Omega^{d-1} M, P_{d}\right) .
\end{aligned}
$$

Since $P_{d}$ is a finitely generated projective $\Lambda$-module,

$$
\operatorname{Ext}_{\Lambda}^{1}\left(\Omega^{d-1} M, P_{d}\right)=\operatorname{Ext}_{\Lambda}^{d}\left(M, P_{d}\right)=0 .
$$

Thus $\operatorname{Hom}_{\Lambda}\left(f_{d}, P_{d}\right)$ is surjective. So there exists a homomorphism $g_{d}$ from $P_{d-1}$ into $P_{d}$ such that the composition $g_{d} \circ f_{d}$ is the identity on $P_{d}$. Therefore the short exact sequence $0 \rightarrow P_{d} \rightarrow P_{d-1} \rightarrow \Omega^{d-1} M \rightarrow 0$ splits, and hence $\Omega^{d-1} M$ is a direct summand of the projective module $P_{d-1}$, which is a contradiction.

Definition 1.3. For a ring $R$ and a subclass $\mathfrak{M}$ of $\operatorname{Mod} R$, we define the assertion $\mathrm{P}_{R}(\mathfrak{M})$ that means that, for any $M \in \mathfrak{M}$, if $\operatorname{Ext}_{\bar{R}}^{\geq 1}(M, R)=0$ then $M$ is projective.

Remark 1.4. For any Noetherian ring $\Lambda$ with finite global dimension and any finitely generated $\Lambda$-module $M$, there is a projective precover of $M$ which is finitely generated. So the above proof works for any Noetherian ring of finite global dimension. Therefore, for any Noetherian ring $\Lambda$ of finite global dimension, $\mathrm{P}_{\Lambda}(\bmod \Lambda)$. 
It is known that any path algebra $K Q$ over an algebraically closed field $K$, even when the quiver $Q$ is not finite, is hereditary, that is, its global dimension is not larger than 1 (see e.g. [14, §8.2]). So any path algebra over an algebraically closed field is an algebra with finite global dimension. Therefore THEOREM 1.2 implies the following.

Corollary 1.5. Suppose that $K$ is an algebraically closed field and $Q$ is a finite acyclic quiver. Then $\mathrm{P}_{K Q}(\bmod K Q)$. In particular, for any finitely generated $K Q$ module $M$, if $\operatorname{Ext}_{K Q}^{1}(M, K Q)=0$ then $M$ is projective.

Remark 1.6. A finite quiver of the form

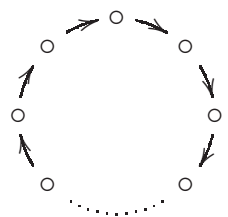

is called a cyclic quiver. Since the path algebra of a cyclic quiver $Q$ over an algebraically closed field $K$ is Noetherian with finite global dimension, it follows from REMARK 1.4 that $\mathrm{P}_{K Q}(\bmod K Q)$.

We can extend the above corollary to some infinite quivers. To introduce such infinite quivers explicitly, we define the following notions.

Definition 1.7. (1) A quiver $P=\left(P_{0}, P_{1}\right)$ is called a subquiver of a quiver $Q=\left(Q_{0}, Q_{1}\right)$ if $P_{0}$ and $P_{1}$ are subsets of $Q_{0}$ and $Q_{1}$ respectively (hence, for any $a \in P_{1}, s(a)$ and $t(a)$ belong to $\left.P_{0}\right)$.

(2) For a quiver $Q$, a subquiver $P$ of $Q$, a field $K$ and a $K$-linear representation $\mathcal{X}$ of $Q$, the $K$-linear representation $\mathcal{X} \uparrow P$ of the quiver $P$ is called the restricted representation of $\mathcal{X}$ by $P$ if for every $v \in P_{0}$ and $a \in P_{1},(\mathcal{X} \uparrow$ $P)_{v}=\mathcal{X}_{v}$ and $(\mathcal{X} \uparrow P)_{a}=\mathcal{X}_{a}$.

(3) For a quiver $Q=\left(Q_{0}, Q_{1}\right)$ and a subset $P_{0}^{\prime}$ of $Q_{0}$, the closure of $P_{0}^{\prime}$ under $Q$ is the subquiver ${\overline{P_{0}^{\prime}}}^{Q}=\left(\left({\overline{P_{0}^{\prime}}}^{Q}\right)_{0},\left({\overline{P_{0}^{\prime}}}^{Q}\right)_{1}\right)$ of the quiver $Q$ such that

$\left({\overline{P_{0}^{\prime}}}^{Q}\right)_{0}:=\left\{v \in Q_{0}:\right.$ there exists a path from a member of $P_{0}^{\prime}$

to the vertex $v$ through the quiver $Q\}$

and

$$
\left({\overline{P_{0}^{\prime}}}^{Q}\right)_{1}:=\left\{a \in Q_{1}: s(a) \in\left({\overline{P_{0}^{\prime}}}^{Q}\right)_{0}\right\} .
$$

A subquiver $P$ of a quiver $Q$ is called a closed subquiver of $Q$ if $P$ is a closure of some subset of $Q_{0}$ under $Q$. A subquiver $P$ of a quiver $Q$ is called a finite closed subquiver of $Q$ if $P$ is a closed subquiver of $Q$ and it is also a finite quiver.

Proposition 1.8. Suppose that $K$ is a field, $Q$ is a quiver, $\mathcal{X}$ is a $K$-linear representation of $Q$ such that $\operatorname{Ext}_{K Q}^{1}(G(\mathcal{X}), K Q)=0$, and $P$ is a closed subquiver of $Q$. Then $\operatorname{Ext}_{K P}^{1}(G(\mathcal{X} \uparrow P), K P)=0$. 
Proof. Let $S$ be the functor from the category $\operatorname{Rep}_{K} Q$ into the category $\operatorname{Rep}_{K} P$ such that, for each $K$-linear representation $\mathcal{Y}$ of $Q, S(\mathcal{Y}):=\mathcal{Y} \uparrow P$, and let $T$ be the functor from $\operatorname{Rep}_{K} P$ into $\operatorname{Rep}_{K} Q$ such that, for each $K$-linear representation $\mathcal{Z}$ of $P, T(\mathcal{Z})$ is the $K$-linear representation of $Q$ such that $T(\mathcal{Z})_{v}:=\mathcal{Z}_{v}$ for every $v \in P_{0}, T(\mathcal{Z})_{v}$ is the trivial $K$-vector space for every $v \in Q_{0} \backslash P_{0}, T(\mathcal{Z})_{a}:=\mathcal{Z}_{a}$ for every $a \in P_{1}$, and $T(\mathcal{Z})_{a}$ is the unique $K$-linear map from the trivial $K$-vector space $T(\mathcal{Z})_{s(a)}$ into the $K$-vector space $T(\mathcal{Z})_{t(a)}$ for every $a \in Q_{1} \backslash P_{1}$. We notice that both $S$ and $T$ are exact functors, and the functor $T$ is a right adjoint of the restricted functor $S$. Moreover, since $P$ is a closed subquiver of $Q, T$ is well-defined, that is, the above $T(\mathcal{Z})$ is certainly a $K$-representation of $Q$. We also notice that the composition $S \circ T$ is the identity functor over $\operatorname{Rep}_{K} P$, and

$$
K P=\bigoplus_{v \in P_{0}} e_{v} K P=\bigoplus_{v \in P_{0}} e_{v} K Q,
$$

which implies that $T(F(K P))$ is a direct summand of $F(K Q)$. Note that $G(T(F(K P)))$ is just $K P$ as a $K Q$-module, so it follows from our assumption that $\operatorname{Ext}_{K Q}^{1}(G(\mathcal{X}), K P)=0$.

$\operatorname{Ext}_{K Q}^{1}(G(\mathcal{X}), K P)=0$ means that any short exact sequence of $K$-linear representations of $Q$ of the form

$$
0 \longrightarrow T(F(K P)) \longrightarrow \mathcal{E} \stackrel{\varphi}{\longrightarrow} \mathcal{X} \longrightarrow 0
$$

splits. We note that in such a short exact sequence, for any $v \in Q_{0} \backslash P_{0}, \mathcal{E}_{v}=\mathcal{X}_{v}$ and $\varphi_{v}$ is an automorphism of $\mathcal{X}_{v}$ (because $T(F(K P))_{v}$ is the trivial $K$-vector space). So, for any short exact sequence of $K$-linear representations of $P$ of the form

$$
L^{\prime}: \quad 0 \longrightarrow F(K P) \longrightarrow \mathcal{E}^{\prime} \longrightarrow \mathcal{X} \uparrow P \longrightarrow 0,
$$

there exists a short exact sequence of $K$-linear representations of $Q$ of the form

$$
L: 0 \longrightarrow T(F(K P)) \longrightarrow \mathcal{E} \longrightarrow \mathcal{X} \longrightarrow 0
$$

such that $S(L)=L^{\prime}$. Therefore, it follows that any short exact sequence of $K$-linear representations of $P$ of the form

$$
0 \longrightarrow F(K P) \longrightarrow \mathcal{E}^{\prime} \longrightarrow \mathcal{X} \uparrow P \longrightarrow 0
$$

splits, which is equivalent to say that $\operatorname{Ext}_{K P}^{1}(G(\mathcal{X} \uparrow P), K P)=0$.

Proposition 1.9. Suppose that $K$ is a field, $Q$ is an acyclic quiver that contains the quiver

$$
v_{0} \longleftarrow v_{1} \longleftarrow v_{2} \longleftarrow \cdots \longleftarrow v_{n} \longleftarrow v_{n+1} \longleftarrow \cdots
$$

as a subquiver, and $\mathcal{X}$ is a finite dimensional $K$-linear representation of $Q$ such that, for each $n \in \omega, \mathcal{X}_{v_{n}} \neq\left\{0_{K}\right\}$, and $\mathcal{X} \uparrow{\overline{\left\{v_{n}\right.}}^{Q}$ is a direct sum of finitely many copies of the corresponding $K$-linear representation $F\left(e_{v_{n}} K Q\right)$ of $e_{v_{n}} K Q$. Then $\operatorname{Ext}_{K Q}^{1}(G(\mathcal{X}), K Q) \neq 0$.

In [9, Definition 3.5], some type of quivers is defined, which is called rooted. It is true that a quiver $Q$ is rooted iff $Q$ does not contain the quiver $A_{\infty}^{\leftarrow}$ as a subquiver 
[9. Proposition 3.6]. So a quiver that satisfies the assumption of the proposition is not rooted. For example, let $Q$ be the following quiver

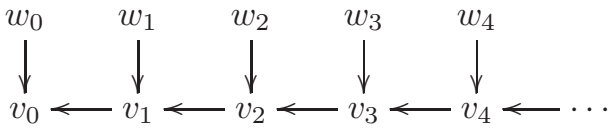

and let $P:={\overline{\left\{v_{n}: n \in \omega\right\}}}^{Q}$. Then the quiver $Q$ is a non-rooted quiver, and the quiver $P$ is different from $Q$, in fact, $P_{0}=\left\{v_{n}: n \in \omega\right\}$. For another example, let $Q$ be the following quiver

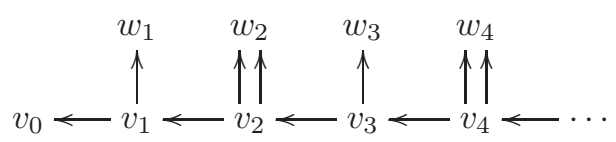

and let $P:={\overline{\left\{v_{n}: n \in \omega\right.}}^{Q}$. Then the quiver $P$ is equal to the quiver $Q$ in this case, and $P_{0} \backslash\left\{v_{n}: n \in \omega\right\}=\left\{w_{n}: n \in \omega\right\}$.

Proof. Let $P:={\overline{\left\{v_{n}: n \in \omega\right\}}}^{Q}$. By Proposition 1.8, it suffices to show that $\operatorname{Ext}_{K P}^{1}(G(\mathcal{X} \uparrow P), K P) \neq 0$. Since

$$
K P=\left(\bigoplus_{n \in \omega} e_{v_{n}} K P\right) \oplus\left(\bigoplus_{v \in P_{0} \backslash\left\{v_{n}: n \in \omega\right\}} e_{v} K P\right)
$$

and

$$
\operatorname{Ext}_{K Q}^{1}\left(M, N_{0} \oplus N_{1}\right)=\operatorname{Ext}_{K Q}^{1}\left(M, N_{0}\right) \oplus \operatorname{Ext}_{K Q}^{1}\left(M, N_{1}\right)
$$

in general, it suffices to show that $\operatorname{Ext}_{K P}^{1}\left(G(\mathcal{X} \uparrow P), \bigoplus_{n \in \omega} e_{v_{n}} K P\right) \neq 0$.

For each $n \in \omega$, let

$$
d_{n}:=\max _{i \in \omega} \mid\left\{p: p \text { is a path from } v_{i} \text { to } v_{n} \text { on } P\right\} \mid,
$$

when such maximum exists as a finite number, or $d_{n}:=\infty$ otherwise. Notice that, for each $n \in \omega$, the dimension of the $K$-vector space $F\left(e_{v_{n}} K Q\right)_{v_{0}}$ is equal the number of paths from $v_{n}$ to $v_{0}$. So, if infinitely many $d_{n}$ were larger than 1 , then the dimension of $\mathcal{X}_{v_{0}}$ had to be infinite. Thus, for all but finitely many $n \in \omega$, $d_{n}=1$. Therefore, without loss of generality we may assume that, for every $n \in \omega$, $d_{n}=1$. Hence there is a $d \in \omega \backslash\{0\}$ such that, for any $n \in \omega$,

$$
\mathcal{X} \uparrow{\overline{\left\{v_{n}\right\}}}^{P}=\bigoplus_{d} F\left(e_{v_{n}} K P\right),
$$

where the last term is the outer direct sum of $d$ many copies of $F\left(e_{v_{n}} K P\right)$. (Notice that $d$ is the dimension of $\mathcal{X}_{v_{n}}$.) For each $n \in \omega$, let $a_{n}$ be the unique arrow from $v_{n+1}$ to $v_{n}$, and, for each $v \in P_{0}$, let

$$
m(v):=\min \left\{m \in \omega: \text { there is a path from } v_{m} \text { to } v\right\} .
$$

Then, for any $v \in P_{0}$ and $n \geq m(v)$, any path from $v_{n}$ to $v$ is of the form $a_{n-1} \cdots a_{m(v)} p^{\prime}$, for some path $p^{\prime}$ from $v_{m(v)}$ to $v$ in $P$. Thus

$$
\mathcal{X} \uparrow P=\bigoplus_{d} \mathcal{X}^{0},
$$


where $\mathcal{X}^{0}$ is the $K$-linear representation of $P$ such that: for each $v \in P_{0}, \mathcal{X}_{v}^{0}$ is the $K$-vector space with basis the set of all paths from $v_{m(v)}$ to $v$; for each $n \in \omega, \mathcal{X}_{a_{n}}^{0}$ is the $K$-linear map from $\mathcal{X}_{v_{n+1}}^{0}$ onto $\mathcal{X}_{v_{n}}^{0}$ such that $\mathcal{X}_{a_{n}}^{0}\left(e_{v_{n+1}}\right)=e_{v_{n}}$; and, for each $a \in P_{1} \backslash\left\{a_{n}: n \in \omega\right\}, \mathcal{X}_{a}^{0}$ is the $K$-linear map from $\mathcal{X}_{s(a)}^{0}$ onto $\mathcal{X}_{t(a)}^{0}$ such that, for each path $p$ from $v_{m(s(a))}$ to $s(a), \mathcal{X}_{a}^{0}(p):=p a$. Since

$$
\operatorname{Ext}_{K P}^{1}\left(\bigoplus_{i \in I} M_{i}, K P\right)=\prod_{i \in I} \operatorname{Ext}_{K P}^{1}\left(M_{i}, K P\right)
$$

in general, it suffices to show that $\operatorname{Ext}_{K P}^{1}\left(G\left(\mathcal{X}^{0}\right), \bigoplus_{n \in \omega} e_{v_{n}} K P\right) \neq 0$.

To see this, let $\pi$ be the canonical $K P$-epimorphism from $\bigoplus_{n \in \omega} e_{v_{n}} K P$ onto $G\left(\mathcal{X}^{0}\right)$ such that, for each $n \in \omega, \pi\left(e_{v_{n}}\right):=e_{v_{n}}$, and, for each $v \in P_{0}$ and each path $p$ in $P$ ending in $v$ of the form $p=a_{n-1} \cdots a_{m(v)} p^{\prime}, \pi(p):=p^{\prime}$. Then $\operatorname{Ker}(\pi)$ is the $K P$-submodule of $\bigoplus_{n \in \omega} e_{v_{n}} K P$ which is generated by the set

$$
\left\{e_{v_{m}}-a_{n} \cdots a_{m}: m, n \in \omega, m \leq n\right\} .
$$

Applying $\operatorname{Hom}_{K P}(-, K P)$ to the exact sequence

$$
0 \rightarrow \operatorname{Ker}(\pi) \stackrel{\operatorname{id}_{\operatorname{Ker}(\pi)}}{\longrightarrow} \bigoplus_{n \in \omega} e_{v_{n}} K P \rightarrow G\left(\mathcal{X}^{0}\right) \rightarrow 0
$$

we obtain the exact sequence

$$
\begin{aligned}
& 0 \rightarrow \operatorname{Hom}_{K P}\left(G\left(\mathcal{X}^{0}\right), K P\right) \rightarrow \\
& \quad \operatorname{Hom}_{K P}\left(\bigoplus_{n \in \omega} e_{v_{n}} K P, K P\right) \stackrel{\operatorname{Hom}_{K P}\left(\operatorname{id}_{\operatorname{Ker}(\pi)}, K P\right)}{\longrightarrow} \operatorname{Hom}_{K P}(\operatorname{Ker}(\pi), K P) .
\end{aligned}
$$

Then

$$
\operatorname{Ext}_{K P}^{1}\left(G\left(\mathcal{X}^{0}\right), \bigoplus_{n \in \omega} e_{v_{n}} K P\right)=\operatorname{Hom}_{K P}(\operatorname{Ker}(\pi), K P) / \operatorname{Im}\left(\operatorname{Hom}_{K P}\left(\operatorname{id}_{\operatorname{Ker}(\pi)}, K P\right)\right) .
$$

For each non-stationary path $b_{n} \cdots b_{0}$ of $P$, we fix the notation $b_{i} \cdots b_{0}$ by induction on $i \leq n$ in such a way that

$$
b_{0} \cdots b_{0}:=b_{0}
$$

and, for $i \leq n$,

$$
b_{i+1} \cdots b_{0}:=b_{i+1} b_{i} \cdots b_{0} .
$$

For each $m, n \in \omega$ with $m \leq n$, define

$$
\varphi\left(e_{v_{m}}-a_{n} \cdots a_{m}\right):=\sum_{i=0}^{n-m} a_{m+i} \cdots a_{m} .
$$


Then, for each $l, m, n \in \omega$ with $l<m \leq n$,

$$
\begin{aligned}
\varphi\left(e_{v_{m}}-a_{n} \cdots a_{m}\right) a_{m-1} \cdots a_{l} & =\left(\sum_{i=0}^{n-m} a_{m+i} \cdots a_{m}\right) a_{m-1} \cdots a_{l} \\
& =\left(\sum_{i=0}^{n-l} a_{l+i} \cdots a_{l}\right)-\left(\sum_{i=0}^{m-1-l} a_{l+i} \cdots a_{l}\right) \\
& =\varphi\left(e_{v_{l}}-a_{n} \cdots a_{l}\right)-\varphi\left(e_{v_{l}}-a_{m-1} \cdots a_{l}\right)
\end{aligned}
$$

and

$$
\begin{aligned}
\left(e_{v_{m}}-a_{n} \cdots a_{m}\right) a_{m-1} \cdots a_{l} & =a_{m-1} \cdots a_{l}-a_{n} \cdots a_{l} \\
& =\left(e_{v_{l}}-a_{n} \cdots a_{l}\right)-\left(e_{v_{l}}-a_{m-1} \cdots a_{l}\right) .
\end{aligned}
$$

Thus, we can extend $\varphi$ to a $K P$-homomorphism from $\operatorname{Ker}(\pi)$ into $K P$. To finish the proof, it is sufficient to show that $\varphi$ is not in $\operatorname{Im}\left(\operatorname{Hom}_{K P}\left(\operatorname{id}_{\operatorname{Ker}(\pi)}, K P\right)\right)$.

Assume it is, and let $\psi \in \operatorname{Hom}_{K P}\left(\bigoplus_{n \in \omega} e_{v_{n}} K P, K P\right)$ be such that

$$
\varphi=\operatorname{Hom}_{K P}\left(\operatorname{id}_{\operatorname{Ker}(\pi)}, K P\right)(\psi)=\psi \uparrow \operatorname{Ker}(\pi) .
$$

For each $n \in \omega$,

$$
\begin{aligned}
\psi\left(e_{v_{0}}\right)-\psi\left(e_{v_{n+2}}\right) a_{n+1} \cdots a_{0} & =\psi\left(e_{v_{0}}\right)-\psi\left(a_{n+1} \cdots a_{0}\right) \\
& =\psi\left(e_{v_{0}}-a_{n+1} \cdots a_{0}\right) \\
& =\sum_{i=0}^{n+1} a_{i} \cdots a_{0} .
\end{aligned}
$$

Therefore, for every $n \in \omega, \psi\left(e_{v_{0}}\right)$ belongs to the set

$$
\left(\sum_{i=0}^{n} a_{i} \cdots a_{0}\right)+K P\left(a_{n+1} \cdots a_{0}\right) .
$$

However, this is a contradiction because $\psi\left(e_{v_{0}}\right)$ have to belong to $K P$.

Theorem 1.10. Suppose that $K$ is an algebraically closed field, and $Q$ is a connected quiver such that, for any finite subset $P_{0}^{\prime}$ of $Q_{0}$, the closure of $P_{0}^{\prime}$ under $Q$ is a finite acyclic quiver. Then $\mathrm{P}_{K Q}\left(\operatorname{rep}_{K} Q\right)$.

For example, the following quivers satisfy the assumption of the theorem:

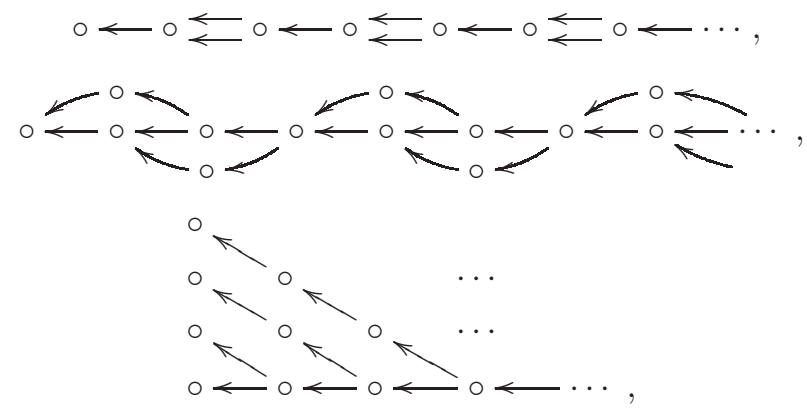




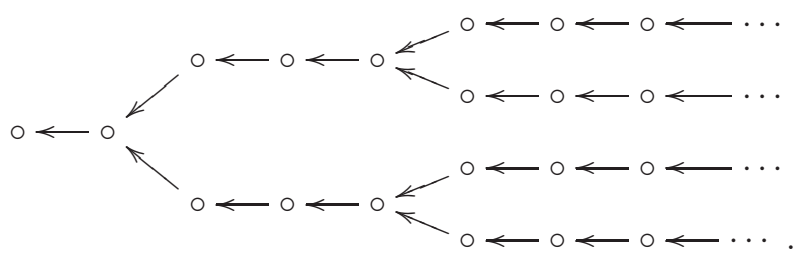

Note that any infinite quiver as in the assumption of the theorem contains at least one of the following quivers as a subquiver:

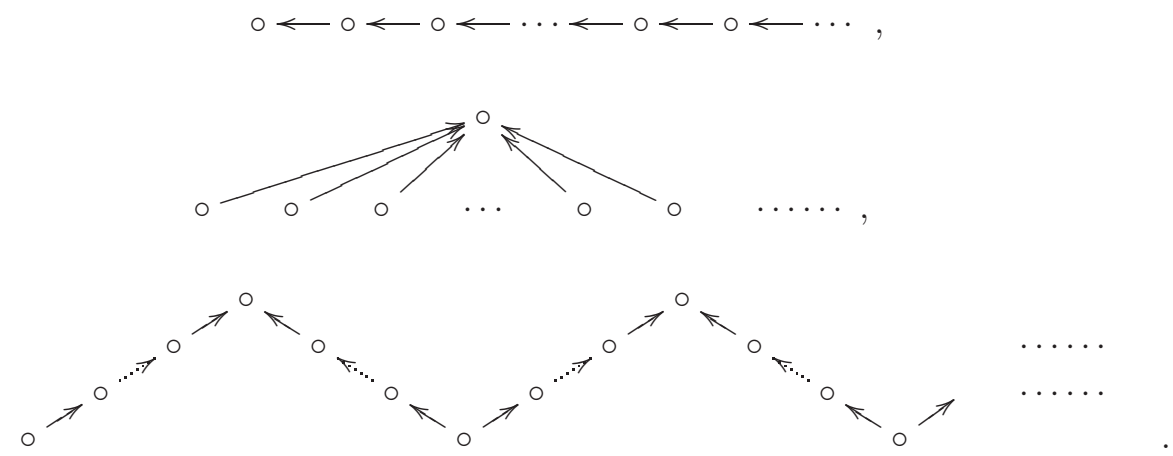

Proof. This theorem has been proved when $Q$ is a finite quiver in Corollary 1.5 Suppose that $Q$ is an infinite quiver, and $\mathcal{X}$ is a finite dimensional $K$-linear representation of $Q$ such that $\operatorname{Ext}_{K Q}^{1}(G(\mathcal{X}), K Q)=0$. We show that $\mathcal{X}$ is projective. Since

$$
\operatorname{Ext}_{K Q}^{1}\left(\bigoplus_{i \in I} M_{i}, K Q\right)=\prod_{i \in I} \operatorname{Ext}_{K Q}^{1}\left(M_{i}, K Q\right)
$$

in general, without loss of generality we may assume that $\mathcal{X}$ is indecomposable.

By Proposition 1.8, for every finite closed subquiver $P$ of $Q, \operatorname{Ext}_{K P}^{1}(G(\mathcal{X} \uparrow$ $P), K P)=0$. Therefore, by $\mathrm{P}_{K P}(\bmod K P), \mathcal{X} \uparrow P$ is projective. It is known that any indecomposable projective $K P$-module is of the form $e_{v} K P$ for some $v \in P_{0}$ [3. §III.2]. Since $P$ is a closed subquiver of $Q$, the underlying set of $e_{v} K P$ is equal to $e_{v} K Q$, and $F\left(e_{v} K Q\right) \uparrow P=F\left(e_{v} K P\right)$. So, since $\mathcal{X}$ is finite dimensional, $\mathcal{X} \uparrow P$ is isomorphic to a direct sum of finitely many $K$-linear representations of the form $F\left(e_{v} K Q\right)$ for $v \in P_{0}$. Therefore, since $\mathcal{X}$ is indecomposable, only one of the following statements hold:

(1) $\mathcal{X}$ is isomorphic to $F\left(e_{v} K Q\right)$ for some $v \in Q_{0}$, or

(2) $Q$ contains the quiver

$$
v_{0} \longleftarrow v_{1} \longleftarrow v_{2} \longleftarrow \cdots \longleftarrow v_{n} \longleftarrow v_{n+1} \longleftarrow \cdots
$$

as a subquiver such that, for each $n \in \omega, \mathcal{X}_{v_{n}} \neq\left\{0_{K}\right\}$, and $\mathcal{X} \uparrow{\overline{\left\{v_{n}\right.}}^{Q}$ is a direct sum of finitely many copies of $F\left(e_{v_{n}} K Q\right)$.

By Proposition 1.9 and the assumption that $\operatorname{Ext}_{K Q}^{1}(G(\mathcal{X}), K Q)=0, \mathcal{X}$ is isomorphic to $F\left(e_{v} K Q\right)$ for some $v \in Q_{0}$. By our assumption, $\overline{\{v\}}^{Q}$ is a finite acyclic quiver, so $e_{v}$ is an idempotent of $K Q$. Hence $e_{v} K Q$ is projective, so is $\mathcal{X}$. 
1.3. Martin's Axiom. Martin's Axiom was introduced by Martin and Solovay [18. This axiom cannot be neither proved nor refuted from axiomatic set theory ZFC, so it is consistent with ZFC. Martin's Axiom can be considered as a generalization of the Baire category theorem (see e.g. [17, Theorem III.4.7]). MA $\mathrm{\aleph}_{\aleph_{1}}$ denotes Martin's Axiom for $\aleph_{1}$ many dense sets. In this paper we use UP*2, which is one combinatorial consequence from $\mathrm{MA}_{\aleph_{1}}$.

Definition 1.11. (1) A ladder system (on $\omega_{1}$ ) is a sequence $\left\langle C_{\alpha}: \alpha \in \omega_{1} \cap \operatorname{Lim}\right\rangle$ such that

- for each $\alpha \in \omega_{1} \cap \operatorname{Lim}, C_{\alpha}$ is a cofinal subset of $\alpha$, that is,

$$
\forall \xi \in \alpha \exists \eta \in C_{\alpha}(\xi \in \eta),
$$

and

- $C_{\alpha}$ is of order type $\omega$, that is, the elements of $C_{\alpha}$ can be enumerated as $\left\{\zeta_{n}^{\alpha}: n \in \omega\right\}$ increasingly, that is, for every $m, n \in \omega$, if $m \in n$, then $\zeta_{m}^{\alpha} \in \zeta_{n}^{\alpha}$.

(2) A coloring $\left\langle d_{\alpha}: \alpha \in \omega_{1} \cap \operatorname{Lim}\right\rangle$ of a ladder system $\left\langle C_{\alpha}: \alpha \in \omega_{1} \cap \operatorname{Lim}\right\rangle$ is a sequence of functions such that the domain of each $d_{\alpha}$ is $C_{\alpha}$.

(3) We say that a function $f$ with domain $\omega_{1}$ uniformizes a coloring $\left\langle d_{\alpha}: \alpha \in\right.$ $\left.\omega_{1} \cap \operatorname{Lim}\right\rangle$ of a ladder system $\left\langle C_{\alpha}: \alpha \in \omega_{1} \cap \operatorname{Lim}\right\rangle, C_{\alpha}=\left\{\zeta_{n}^{\alpha}: n \in \omega\right\}$, if for every $\alpha \in \omega_{1} \cap \operatorname{Lim}$, the restricted function $f\left\lceil C_{\alpha}\right.$ of $f$ by $C_{\alpha}$ is equal to the function $d_{\alpha}$ for all but finitely many points, that is, there exists an $N \in \omega$ such that, for any $n \in \omega \backslash N, f\left(\zeta_{n}^{\alpha}\right)=d_{\alpha}\left(\zeta_{n}^{\alpha}\right)$.

(4) The assertion UP means that, for any sequence $\left\langle X_{\beta}: \beta \in \omega_{1}\right\rangle$ of countable sets and any coloring $\left\langle d_{\alpha}: \alpha \in \omega_{1} \cap \operatorname{Lim}\right\rangle$ of a ladder system $\left\langle C_{\alpha}: \alpha \in \omega_{1} \cap \operatorname{Lim}\right\rangle$, whenever $d_{\alpha}\left(\zeta_{n}^{\alpha}\right)$ belongs to $X_{\zeta_{n}^{\alpha}}$ for any $\alpha \in \omega_{1} \cap \operatorname{Lim}$ and $n \in \omega$, there exists a function with domain $\omega_{1}$ which uniformizes the coloring $\left\langle d_{\alpha}: \alpha \in \omega_{1} \cap \operatorname{Lim}\right\rangle$.

Theorem 1.12 (Devlin-Shelah [6, Theorem 5.2]). MA $_{\aleph_{1}}$ implies UP.

The assertion UP was inspired by Shelah's proof that $\mathrm{MA}_{\aleph_{1}}$ implies the existence of a non-free Whitehead group [20, Theorem 3.5] (see also [10]).

1.4. Trlifaj's construction. In this paper, our modules are built by modifying Trlifaj's construction. As every proof in $\$ 2$ is fairly self-contained, the reader does not need to be familiar with this construction. Trlifaj's construction is a quotient module of the outer direct sum $\bigoplus_{\xi \in \omega_{1}} F^{\xi}$ of some sequence $\left\langle F^{\xi}: \xi \in \omega_{1}\right\rangle$ of modules, defined in [22, Definition 1.1] and [15, Notation 5.3], which seems to be inspired by Shelah's solution of Whitehead Problem 20. To fix our notation and understand our construction better, Trlifaj's construction is presented as follows.

Let $R$ be a ring with identity and let

$$
F_{0} \stackrel{f_{0}}{\longrightarrow} F_{1} \stackrel{f_{1}}{\longrightarrow} \cdots \stackrel{f_{n-1}}{\longrightarrow} F_{n} \stackrel{f_{n}}{\longrightarrow} F_{n+1} \stackrel{f_{n+1}}{\longrightarrow} \cdots
$$

be a countable direct system of $R$-modules. Let $\left\langle C_{\alpha}: \alpha \in \omega_{1} \cap \operatorname{Lim}\right\rangle$ be a ladder system such that

$$
C_{\alpha}=\left\{\zeta_{n}^{\alpha}: n \in \omega\right\}
$$

\footnotetext{
${ }^{* 2}$ This notation follows 11 but it is not that common in set theory.
} 
is an increasing enumeration and assume that, for each $n \in \omega, \zeta_{n}^{\alpha}$ is of the form $\delta+n+1$ for some $\delta \in \alpha \cap(\{0\} \cup \operatorname{Lim})$ (there is such a ladder system). Define $F^{0}:=\left\{0_{R}\right\}$; for each $\gamma \in \omega_{1} \backslash \operatorname{Lim}$ with $\gamma=\delta+n_{\gamma}+1$ for some $\delta \in \gamma \cap(\{0\} \cup \operatorname{Lim})$ and $n_{\gamma} \in \omega$, define $F^{\gamma}:=F_{n_{\gamma}}$; and, for each $\delta \in \omega_{1} \cap \operatorname{Lim}$, define $F^{\delta}:=\bigoplus_{n \in \omega} F_{n}$. So, for each member $x$ of the outer direct sum $\bigoplus_{\xi \in \omega_{1}} F^{\xi}, x$ forms a finite support function with domain included in $\omega_{1}$ and, for each $\alpha \in \omega_{1}, x(\alpha)$ belongs to $F^{\alpha}$. Hence, if $\delta \in \omega_{1} \cap \operatorname{Lim}$, then $x(\delta)$ belongs to the outer direct sum $F^{\delta}:=\bigoplus_{n \in \omega} F_{n}$, which also forms a finite support function with domain included in $\omega$. For each $\delta \in \omega_{1} \cap$ Lim, define the $R$-submodule

$$
\begin{aligned}
& G_{\delta}:=\left\langle\left\{ x \in \bigoplus_{\xi \in \omega_{1}} F^{\xi}: \text { for some } n \in \omega, \operatorname{supp}(x)=\left\{\zeta_{n}^{\delta}, \delta\right\}\right.\right. \\
& \operatorname{supp}(x(\delta))=\{n, n+1\}, x\left(\zeta_{n}^{\delta}\right)=x(\delta)(n), \\
&\text { and } \left.\left.x(\delta)(n+1)=f_{n}(x(\delta)(n))\right\}\right\rangle_{R}
\end{aligned}
$$

of the $R$-module $\bigoplus_{\xi \in \omega_{1}} F^{\xi}$, and define

$$
I_{\omega_{1}}:=\sum_{\delta \in \omega_{1} \cap \operatorname{Lim}} G_{\delta}
$$

which is an $R$-submodule of the $R$-module $\bigoplus_{\xi \in \omega_{1}} F^{\xi}$. Trlifaj's construction is the quotient $R$-module $\bigoplus_{\xi \in \omega_{1}} F^{\xi} / I_{\omega_{1}}$ of the $R$-module $\bigoplus_{\xi \in \omega_{1}} F^{\xi}$ by the $R$-submodule $I_{\omega_{1}}$. Trlifaj applied this construction for a non-left perfect ring [22], and HerberaTrlifaj applied it to analyze some classes of modules called Kaplansky classes or deconstructible classes [15. For further properties of this module, see [15, §5].

\section{Some infinitely Generated MOdules of PATH ALGEBras}

Throughout this section, we fix a ladder system $\left\langle C_{\alpha}: \alpha \in \omega_{1} \cap \operatorname{Lim}\right\rangle$ such that

$$
C_{\alpha}=\left\{\zeta_{n}^{\alpha}: n \in \omega\right\}
$$

is an increasing enumeration and, for each $n \in \omega, \zeta_{n}^{\alpha}$ is of the form $\delta+n+1$ for some $\delta \in \alpha \cap(\{0\} \cup \operatorname{Lim})$. We note that, for any $\alpha, \beta \in \omega_{1} \cap \operatorname{Lim}$ and $m, n \in \omega$, if $\zeta_{m}^{\alpha}=\zeta_{n}^{\beta}$ then $m=n$. For $\gamma \in \omega_{1} \backslash(\{0\} \cup \operatorname{Lim})$, let $n_{\gamma} \in \omega$ be the unique integer such that $\gamma=\delta+n_{\gamma}+1$ for some (unique) $\delta \in \omega_{1} \cap(\{0\} \cup \operatorname{Lim})$.

For each subsection of this section, we deal with some quiver $Q$ and build a non-projective $K Q$-module $M_{\omega_{1}}$. For each quiver $Q$ in each subsection, we use the following notation. For each $v \in Q_{0}, e_{v}$ denotes the path of length 0 from the vertex $v$ (to itself). For $\gamma \in \omega_{1} \backslash \operatorname{Lim}, \alpha \in \omega_{1} \cap \operatorname{Lim}$ and $n \in \omega$, let $F^{\gamma}=F^{\alpha, n}:=K Q$, and let $F^{\alpha}$ be the outer direct sum $\bigoplus_{n \in \omega} F^{\alpha, n}$. For $\gamma \in \omega_{1} \backslash$ Lim and $v \in Q_{0}$, let $e_{v}^{\gamma}$ be 
the member of the outer direct sum $\bigoplus_{\xi \in \omega_{1}} F^{\xi}$ of $K Q$-modules such that

$$
\operatorname{supp}\left(e_{v}^{\gamma}\right)=\{\gamma\}, \quad e_{v}^{\gamma}(\gamma)=e_{v}
$$

For $\alpha \in \omega_{1} \cap \operatorname{Lim}, n \in \omega$ and $v \in Q_{0}$, let $e_{v}^{\alpha, n} \in \bigoplus_{\xi \in \omega_{1}} F^{\xi}$ be such that

$$
\operatorname{supp}\left(e_{v}^{\alpha, n}\right)=\{\alpha\}, \quad \operatorname{supp}\left(e_{v}^{\alpha, n}(\alpha)\right)=\{n\}, \quad e_{v}^{\alpha, n}(\alpha)(n)=e_{v} .
$$

Remark 2.1. The set

$$
\left\{e_{v}^{\gamma}, e_{v}^{\alpha, n}: \gamma \in \omega_{1} \backslash \operatorname{Lim}, v \in Q_{0}, \alpha \in \omega_{1} \cap \operatorname{Lim}, n \in \omega\right\}
$$

is linearly independent with respect to $K Q$ in $\bigoplus_{\xi \in \omega_{1}} F^{\xi}$.

2.1. On a quiver of $A_{\infty}$ type. Throughout this subsection, let $K$ be a field, and $Q$ the quiver $A_{\infty}^{\leftarrow}$ as follows:

$$
0 \stackrel{a_{0}}{\longleftarrow} 1 \stackrel{a_{1}}{\longleftarrow} \ldots \stackrel{a_{n-1}}{\longleftarrow} n \stackrel{a_{n}}{\longleftarrow} n+1 \stackrel{a_{n+1}}{\longleftarrow} \cdots,
$$

that is, the set $Q_{0}$ of vertices is the set of all non-negative integers and the set $Q_{1}$ of arrows is defined by

$$
\left\{n \stackrel{a_{n}}{\longleftarrow} n+1: n \in \omega\right\} .
$$

Since $Q_{0}$ is infinite, $K Q$ does not have an identity. By simplifying the notation in this subsection, for each $\alpha \in \omega_{1} \cap \operatorname{Lim}$ and $n \in \omega$,

$$
e_{n}^{\alpha}:=e_{n}^{\alpha, 0} .
$$

For each $\alpha \in \omega_{1} \cap$ Lim, define

$$
\begin{gathered}
G_{\alpha}:=\left\langle\left\{e_{n}^{\zeta_{n}^{\alpha}}-e_{n}^{\alpha}+e_{n+1}^{\alpha} a_{n}: n \in \omega\right\}\right\rangle_{K Q}, \\
I_{\omega_{1}}:=\sum_{\xi \in \omega_{1} \cap \text { Lim }} G_{\xi} .
\end{gathered}
$$

For each $x \in \bigoplus_{\xi \in \omega_{1}} F^{\xi}, x+I_{\omega_{1}}$ denotes the equivalence class of $x$ in the quotient module $\bigoplus_{\xi \in \omega_{1}} F^{\xi} / I_{\omega_{1}}$. For each $\xi \in \omega_{1}+1$, define the $K Q$-module $M_{\xi}$ by $\left\langle\left\{e_{n_{\gamma}}^{\gamma}+I_{\omega_{1}}: \gamma \in \xi \backslash \operatorname{Lim}\right\} \cup\left\{e_{n}^{\alpha}+I_{\omega_{1}}: \alpha \in \xi \cap \operatorname{Lim}, n \in \omega\right\}\right\rangle_{K Q}$,

which is considered as a $K Q$-submodule of the quotient module $\bigoplus_{\xi \in \omega_{1}} F^{\xi} / I_{\omega_{1}}$.

Remark 2.2. The set $\left\{e_{n}^{\zeta_{n}^{\alpha}}-e_{n}^{\alpha}+e_{n+1}^{\alpha} a_{n}: \alpha \in \omega_{1} \cap \operatorname{Lim}, n \in \omega\right\}$ is linearly independent with respect to $K Q$ in $\bigoplus_{\xi \in \omega_{1}} F^{\xi}$.

In this paper, $\bigoplus_{\omega_{1}} K Q$ denotes the outer direct sum of $\omega_{1}$ many copies of $K Q$, which is considered as a $K Q$-module. 
Claim 2.3. $\operatorname{Ext}_{K Q}^{1}\left(M_{\omega_{1}}, \bigoplus_{\omega_{1}} K Q\right) \neq 0$. In particular, $M_{\omega_{1}}$ is not a projective $K Q$-module.

Proof. $F_{\omega_{1}}$ denotes the $K Q$-module

$$
\left\langle\left\{e_{n_{\gamma}}^{\gamma}: \gamma \in \omega_{1} \backslash \operatorname{Lim}\right\} \cup\left\{e_{n}^{\alpha}: \alpha \in \omega_{1} \cap \operatorname{Lim}, n \in \omega\right\}\right\rangle_{K Q} .
$$

Applying $\operatorname{Hom}_{K Q}\left(-, \bigoplus_{\omega_{1}} K Q\right)$ to the exact sequence

$$
0 \rightarrow I_{\omega_{1}} \stackrel{\operatorname{id}_{I_{\omega_{1}}}}{\longrightarrow} F_{\omega_{1}} \rightarrow M_{\omega_{1}} \rightarrow 0
$$

we obtain the exact sequence

$$
\begin{aligned}
0 \rightarrow \operatorname{Hom}_{K Q}\left(M_{\omega_{1}}, \bigoplus_{\omega_{1}} K Q\right) \rightarrow \\
\operatorname{Hom}_{K Q}\left(F_{\omega_{1}}, \bigoplus_{\omega_{1}} K Q\right) \stackrel{\operatorname{Hom}_{K Q}\left(\operatorname{id}_{I_{1}}, \underset{\omega_{1}}{\bigoplus K Q)} \longrightarrow\right.}{\longrightarrow} \operatorname{Hom}_{K Q}\left(I_{\omega_{1}}, \bigoplus_{\omega_{1}} K Q\right) .
\end{aligned}
$$

Then

$$
\operatorname{Ext}_{K Q}^{1}\left(M_{\omega_{1}}, \bigoplus_{\omega_{1}} K Q\right)=\operatorname{Hom}_{K Q}\left(I_{\omega_{1}}, \bigoplus_{\omega_{1}} K Q\right) / \operatorname{Im}\left(\operatorname{Hom}_{K Q}\left(\operatorname{id}_{I_{\omega_{1}}}, \bigoplus_{\omega_{1}} K Q\right)\right) .
$$

By REMARK 2.2, we can find a $K Q$-homomorphism $\varphi$ in $\operatorname{Hom}_{K Q}\left(I_{\omega_{1}}, \bigoplus_{\omega_{1}} K Q\right)$ such that for each $\alpha \in \omega_{1} \cap \operatorname{Lim}$ and $n \in \omega$,

$$
\varphi\left(e_{n}^{\zeta_{n}^{\alpha}}-e_{n}^{\alpha}+e_{n+1}^{\alpha} a_{n}\right):=e_{n}^{\alpha} .
$$

We show that $\varphi$ does not belong to $\operatorname{Im}\left(\operatorname{Hom}_{K Q}\left(\operatorname{id}_{I_{\omega_{1}}}, \bigoplus_{\omega_{1}} K Q\right)\right)$.

Assume that $\varphi \in \operatorname{Im}\left(\operatorname{Hom}_{K Q}\left(\operatorname{id}_{I_{\omega_{1}}}, \bigoplus_{\omega_{1}} K Q\right)\right)$, and let $\psi$ be a homomorphism in $\operatorname{Hom}_{K Q}\left(F_{\omega_{1}}, \bigoplus_{\omega_{1}} K Q\right)$ such that

$$
\operatorname{Hom}_{K Q}\left(\operatorname{id}_{I_{\omega_{1}}}, \bigoplus_{\omega_{1}} K Q\right)(\psi)=\psi \circ \operatorname{id}_{I_{\omega_{1}}}=\psi \uparrow I_{\omega_{1}}=\varphi .
$$

We note that for each $\gamma \in \omega_{1}$ and $n \in \omega, \operatorname{supp}\left(\psi\left(e_{n}^{\gamma}\right)\right)$ is a finite subset of $\omega_{1}$. So we can take an $\alpha \in \omega_{1} \cap \operatorname{Lim}$ such that, for every $\gamma \in \alpha$ and $n \in \omega, \operatorname{supp}\left(\psi\left(e_{n}^{\gamma}\right)\right)$ is a finite subset of $a * 3$. For each $n \in \omega$,

$$
\psi\left(e_{n}^{\zeta_{n}^{\alpha}}\right)-\psi\left(e_{n}^{\alpha}\right)+\psi\left(e_{n+1}^{\alpha}\right) a_{n}=\psi\left(e_{n}^{\zeta_{n}^{\alpha}}-e_{n}^{\alpha}+e_{n+1}^{\alpha} a_{n}\right)=e_{n}^{\alpha} .
$$

\footnotetext{
${ }^{* 3}$ This can be done by e.g. [17 Exercise III.6.20]
} 
Therefore, by induction on $n \in \omega$,

$$
\begin{aligned}
\psi\left(e_{0}^{\alpha}\right)= & \psi\left(e_{0}^{\zeta_{0}^{\alpha}}\right)-e_{0}^{\alpha}+\psi\left(e_{1}^{\alpha}\right) a_{0} \\
= & \psi\left(e_{0}^{\zeta_{0}^{\alpha}}\right)-e_{0}^{\alpha}+\left(\psi\left(e_{1}^{\zeta_{1}^{\alpha}}\right)-e_{1}^{\alpha}+\psi\left(e_{2}^{\alpha}\right) a_{1}\right) a_{0} \\
= & \psi\left(e_{0}^{\zeta_{0}^{\alpha}}\right)-e_{0}^{\alpha}+\psi\left(e_{1}^{\zeta_{1}^{\alpha}}\right) a_{0}-e_{1}^{\alpha} a_{0}+\psi\left(e_{2}^{\alpha}\right) a_{1} a_{0} \\
= & \cdots \\
= & \psi\left(e_{0}^{\zeta_{0}^{\alpha}}\right)-e_{0}^{\alpha}+\sum_{i=1}^{n} \psi\left(e_{i}^{\zeta_{i}^{\alpha}}\right) a_{i-1} \cdots a_{0}-\sum_{i=1}^{n} e_{i}^{\alpha} a_{i-1} \cdots a_{0} \\
& +\psi\left(e_{n+1}^{\alpha}\right) a_{n} \cdots a_{0} .
\end{aligned}
$$

Hence, for every $n \in \omega$, since each $\operatorname{supp}\left(\psi\left(e_{i}^{\zeta_{i}^{\alpha}}\right)\right)$ does not contain $\alpha$ as a member,

$$
\psi\left(e_{0}^{\alpha}\right)(\alpha) \notin K Q_{\leq n},
$$

where $K Q_{\leq n}$ is the $K$-subspace of $K Q$ generated by all paths of length $\leq n$. This is a contradiction.

The following is similar to [11, Theorem XII 2.2, Proposition XIII 0.2].

Theorem 2.4. Suppose that $K$ is a countable field. Then UP implies that $\operatorname{Ext}_{K Q}^{1}\left(M_{\omega_{1}}, K Q\right)=0$. In particular, $\mathrm{P}_{K Q}(\operatorname{Mod} K Q)$ fails.

Proof. Applying $\operatorname{Hom}_{K Q}(-, K Q)$ to the exact sequence

$$
0 \rightarrow I_{\omega_{1}} \stackrel{\operatorname{id}_{\omega_{\omega_{1}}}}{\longrightarrow} F_{\omega_{1}} \rightarrow M_{\omega_{1}} \rightarrow 0
$$

we obtain the exact sequence

$$
\begin{aligned}
0 \rightarrow \operatorname{Hom}_{K Q}\left(M_{\omega_{1}}, K Q\right) \rightarrow \\
\quad \operatorname{Hom}_{K Q}\left(F_{\omega_{1}}, K Q\right) \stackrel{\operatorname{Hom}_{K Q}\left(\operatorname{id}_{I_{\omega_{1}}}, K Q\right)}{\longrightarrow} \operatorname{Hom}_{K Q}\left(I_{\omega_{1}}, K Q\right) .
\end{aligned}
$$

Then

$$
\operatorname{Ext}_{K Q}^{1}\left(M_{\omega_{1}}, K Q\right)=\operatorname{Hom}_{K Q}\left(I_{\omega_{1}}, K Q\right) / \operatorname{Im}\left(\operatorname{Hom}_{K Q}\left(\operatorname{id}_{I_{\omega_{1}}}, K Q\right)\right) .
$$

Let $\varphi \in \operatorname{Hom}_{K Q}\left(I_{\omega_{1}}, K Q\right)$. We show that $\varphi$ belongs to $\operatorname{Im}\left(\operatorname{Hom}_{K Q}\left(\operatorname{id}_{I_{\omega_{1}}}, K Q\right)\right)$. For each $\alpha \in \omega_{1} \cap \operatorname{Lim}$ and $n \in \omega$, define

$$
d_{\alpha}\left(\zeta_{n}^{\alpha}\right):=\varphi\left(e_{n}^{\zeta_{n}^{\alpha}}-e_{n}^{\alpha}+e_{n+1}^{\alpha} a_{n}\right) .
$$

We notice that, for each $n \in \omega$,

$$
\varphi\left(e_{n}^{\zeta_{n}^{\alpha}}-e_{n}^{\alpha}+e_{n+1}^{\alpha} a_{n}\right) e_{n}=\varphi\left(e_{n}^{\zeta_{n}^{\alpha}}-e_{n}^{\alpha}+e_{n+1}^{\alpha} a_{n}\right),
$$

and, for any $m \in Q_{0} \backslash\{n\}$,

$$
\varphi\left(e_{n}^{\zeta_{n}^{\alpha}}-e_{n}^{\alpha}+e_{n+1}^{\alpha} a_{n}\right) e_{m}=\varphi\left(0 \underset{\xi \in \omega_{1}}{\oplus} F \xi\right)=0_{K Q} .
$$

Thus, for each $n \in \omega, d_{\alpha}\left(\zeta_{n}^{\alpha}\right)$ belongs to the countable set

$$
\sum_{p: \text { path in } Q \text { ending in } n} K p .
$$


Therefore, by UP, we can find a uniformization $f$ of the ladder system coloring $\left\langle d_{\alpha}: \alpha \in \omega_{1} \cap \operatorname{Lim}\right\rangle$, that is, for each $\alpha \in \omega_{1} \cap \operatorname{Lim}$, there is an $N_{\alpha} \in \omega$ such that, for every $n \geq N_{\alpha}, f\left(\zeta_{n}^{\alpha}\right)=d_{\alpha}\left(\zeta_{n}^{\alpha}\right)$.

For each $\alpha \in \omega_{1} \cap \operatorname{Lim}$ and $n \in \omega$, define

- $\psi\left(e_{n}^{\zeta_{n}^{\alpha}}\right):=f\left(\zeta_{n}^{\alpha}\right)$

- $\psi\left(e_{n}^{\alpha}\right):=0_{K Q}$ when $n \geq N_{\alpha}$, and

- by downward induction on $n<N_{\alpha}$, define

$$
\psi\left(e_{n}^{\alpha}\right):=\psi\left(e_{n}^{\zeta_{n}^{\alpha}}\right)+\psi\left(e_{n+1}^{\alpha}\right) a_{n}-\varphi\left(e_{n}^{\zeta_{n}^{\alpha}}-e_{n}^{\alpha}+e_{n+1}^{\alpha} a_{n}\right) .
$$

By Remark 2.1 $\psi$ can be extended to a $K Q$-homomorphism from $F_{\omega_{1}}$ into $K Q$. Therefore

$$
\psi \uparrow I_{\omega_{1}}=\psi \circ \operatorname{id}_{I_{\omega_{1}}}=\operatorname{Hom}_{K Q}\left(\operatorname{id}_{I_{\omega_{1}}}, K Q\right)(\psi)=\varphi,
$$

which finishes the proof.

Remark 2.5. By a similar argument to the one in the previous theorem, it can be proved that if $K$ is a countable field and UP holds, then $\operatorname{Ext}_{K Q}^{1}\left(M_{\omega_{1}}, \bigoplus_{\omega} K Q\right)=0$.

Remark 2.6. By a similar argument as in [10, Lemma 4.3], we can show that if $K$ is a countable field, then $\diamond$ implies $\operatorname{Ext}_{K Q}^{1}\left(M_{\omega_{1}}, K Q\right) \neq 0$. The main ingredient to prove this is the following fact.

Claim 2.7. Suppose that $\operatorname{Ext}_{K Q}\left(M_{\alpha+1} / M_{\alpha}, K Q\right) \neq 0$, and let

$$
0 \longrightarrow K Q \rightarrow C_{\alpha} \stackrel{\pi}{\longrightarrow} M_{\alpha} \longrightarrow 0
$$

be a short exact sequence that splits, that is, there exists a homomorphism $\rho$ from $M_{\alpha}$ into $C_{\alpha}$ such that $\pi \circ \rho=\operatorname{id}_{M_{\alpha}}$. Then there exists a short exact sequence

$$
0 \longrightarrow K Q \rightarrow C_{\alpha+1} \stackrel{\pi^{\prime}}{\longrightarrow} M_{\alpha+1} \longrightarrow 0
$$

such that

$$
\pi^{\prime} \uparrow C_{\alpha}=\pi
$$

and there is no homomorphism $\rho^{\prime}$ from $M_{\alpha+1}$ into $C_{\alpha+1}$ such that

$$
\pi^{\prime} \circ \rho^{\prime}=\operatorname{id}_{M_{\alpha}+1} \quad \text { and } \quad \rho^{\prime} \uparrow M_{\alpha}=\rho .
$$

Since $K Q$ is countable and Claim 2.3 holds, a similar argument as in 10, Theorem 6.3] works well to show that $\operatorname{Ext}_{K Q}\left(M_{\omega_{1}}, K Q\right) \neq 0$. Moreover, by a similar argument as in [16], we can show that, if $K$ is a countable field and there is a set $\left\{S_{\alpha}: \alpha \in \omega_{1}\right\}$ of pairwise disjoint stationary subsets of $\omega_{1}$ such that $\nabla_{S_{\alpha}}$ holds for each $\alpha \in \omega_{1}$, then the cardinality of $\operatorname{Ext}_{K Q}\left(M_{\omega_{1}}, K Q\right)$ is greater than $\aleph_{1}$.

${ }^{* 4} \diamond$ is one set theoretic axiom consistent with ZFC, see e.g. [17. 
2.2. On a circular quiver. In this subsection, let $K$ be a field and $Q$ the following quiver.

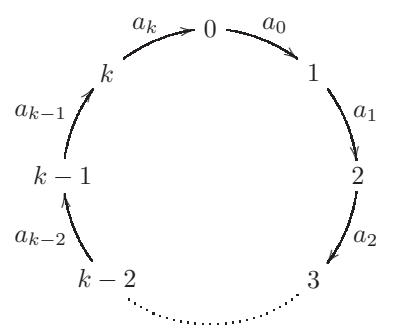

Then the path $a_{0} a_{1} \cdots a_{k}$ is a path in $Q$ whose source and target are both the vertex 0 . We denote the path

$$
\left(a_{0} a_{1} \cdots a_{k}\right)^{0}=e_{0}
$$

and, for each $n \in \omega$, define the path

$$
\left(a_{0} a_{1} \cdots a_{k}\right)^{n+1}=\left(a_{0} a_{1} \cdots a_{k}\right)^{n} a_{0} a_{1} \cdots a_{k} .
$$

Recall that $\sum_{v \in Q_{0}} e_{v}$ is the identity of $K Q$. For each $\alpha \in \omega_{1} \cap$ Lim, define

$$
\begin{gathered}
G_{\alpha}:=\left\langle\left\{e_{0}^{\zeta_{n}^{\alpha}}-e_{0}^{\alpha, n}+e_{0}^{\alpha, n+1} a_{0} a_{1} \cdots a_{k}: n \in \omega\right\}\right\rangle_{K Q}, \\
I_{\omega_{1}}:=\sum_{\xi \in \omega_{1} \cap \operatorname{Lim}} G_{\xi},
\end{gathered}
$$

and, for each $\xi \in \omega_{1}+1$, define the $K Q$-module $M_{\xi}$ by

$$
\left\langle\left\{e_{0}^{\gamma}+I_{\omega_{1}}: \gamma \in \xi \backslash \operatorname{Lim}\right\} \cup\left\{e_{0}^{\alpha, n}+I_{\omega_{1}}: \alpha \in \xi \cap \operatorname{Lim}, n \in \omega\right\}\right\rangle_{K Q},
$$

which is considered as a $K Q$-submodule of the quotient module $\bigoplus_{\xi \in \omega_{1}} F^{\xi} / I_{\omega_{1}}$.

Claim 2.8. $\operatorname{Ext}_{K Q}^{1}\left(M_{\omega_{1}}, \bigoplus_{\omega_{1}} K Q\right) \neq 0$. Therefore, $M_{\omega_{1}}$ is not projective.

Proof. This can be proved in a similar way as in Claim 2.3. To see this, it suffices to replace the formula

$$
\varphi\left(e_{n}^{\zeta_{n}^{\alpha}}-e_{n}^{\alpha}+e_{n+1}^{\alpha} a_{n}\right):=e_{n}^{\alpha}
$$

by the formula

$$
\varphi\left(e_{0}^{\zeta_{n}^{\alpha}}-e_{0}^{\alpha, n}+e_{0}^{\alpha, n+1} a_{0} a_{1} \cdots a_{k}\right):=e_{0}^{\alpha, n}
$$

in the proof of CLAIM 2.3 .

Moreover, by a similar proof as THEOREM 2.4, the following theorem can be proved.

Theorem 2.9. Suppose that $K$ is a countable field. Then UP implies that $\operatorname{Ext}_{K Q}^{1}\left(M_{\omega_{1}}, K Q\right)=0$. In particular, $\mathrm{P}_{K Q}(\operatorname{Mod} K Q)$ fails.

Remark 2.10. As in REMARK 2.6] if $K$ is a countable field and $\diamond$ holds, then $\operatorname{Ext}_{K Q}^{1}\left(M_{\omega_{1}}, K Q\right) \neq 0$. 


\subsection{Generalizations.}

Theorem 2.11. Suppose that $K$ is a countable field and $Q^{\prime}$ is a quiver that contains a subquiver $Q$ of one of the following types

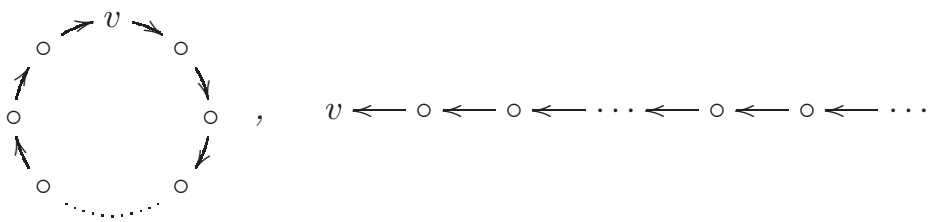

in such a way that the set of all paths in $Q^{\prime}$ ending in $v$ is countable. Then UP implies the failure of $\mathrm{P}_{K Q^{\prime}}\left(\operatorname{Mod} K Q^{\prime}\right)$.

Proof. Let $M_{\omega_{1}}$ be one of the $K Q$-modules constructed before. Then, $M_{\omega_{1}}$ can be considered as a $K Q^{\prime}$-module and, by a similar argument as before, it can be proved that $\operatorname{Ext}_{K Q^{\prime}}^{1}\left(M_{\omega_{1}}, \bigoplus_{\omega_{1}} K Q^{\prime}\right) \neq 0$, and that UP implies $\operatorname{Ext}_{K Q^{\prime}}^{1}\left(M_{\omega_{1}}, K Q^{\prime}\right)=0$.

Acknowledgement. The authors thank Hiroyuki Minamoto, Izuru Mori and Kenta Ueyama for useful comments about this research. Especially, they provided us advice and information about Theorem 1.2. Remark 1.4 and Proposition 1.8 ,

The authors also appreciate the useful comments from the referee.

\section{REFERENCES}

[1] F. W. Anderson and K.R. Fuller. Rings and categories of modules. Second edition. Graduate Texts in Mathematics, 13. Springer-Verlag, New York, 1992.

[2] M. Auslander, I Reiten and S. Smalø. Representation theory of Artin algebras.Cambridge Studies in Advanced Mathematics, 36. Cambridge University Press, Cambridge, 1995.

[3] I. Assem, D. Simson and A. Skowroński. Elements of the representation theory of associative algebras. Vol. 1. Techniques of representation theory. London Mathematical Society Student Texts, 65. Cambridge University Press, Cambridge, 2006.

[4] D. J. Benson. Representations and cohomology. I. Basic representation theory of finite groups and associative algebras. Cambridge Studies in Advanced Mathematics, 30. Cambridge University Press, Cambridge, 1991.

[5] H. Brune. Some left pure semisimple ringoids which are not right pure semisimple. Comm. Algebra 7 (1979), no. 17, 1795-1803.

[6] K. Devlin and S. Shelah. A weak version of $\diamond$ which follows from $2^{\aleph_{0}}<2^{\aleph_{1}}$. Israel J. Math. 29 (1978), no. 2-3, 239-247.

[7] E. E. Enochs and S. Estrada. Projective representations of quivers. (English summary) Comm. Algebra 33 (2005), no. 10,3467-3478.

[8] E. E. Enochs, S. Estrada, J. R. García Rozas and L. Oyonarte. Flat covers of representations of the quiver $A_{\infty}$. Int. J. Math. Math. Sci. 2003, no. 70,4409-4419.

[9] E. E. Enochs, L. Oyonarte and B. Torrecillas. Flat covers and flat representations of quivers. Comm. Algebra 32 (2004), no. 4,1319-1338.

[10] P. C. Eklof. Whitehead's problem is undecidable. Amer. Math. Monthly 83 (1976), no. 10, $775-788$.

[11] P. C. Eklof and A. H. Mekler. Almost free modules. Set-theoretic methods. Revised edition. North-Holland Mathematical Library, 65. North-Holland Publishing Co., Amsterdam, 2002.

[12] D. H. Fremlin. Consequences of Martin's axiom. Cambridge Tracts in Mathematics, 84. Cambridge University Press, Cambridge, 1984.

[13] P. Gabriel, Auslander-Reiten sequences and representation-finite algebras, Representation theory, I (Proc. Workshop, Carleton Univ., Ottawa, Ont., 1979), 1-71, Lecture Notes in Math., 831, Springer, Berlin, 1980. 
[14] P. Gabriel and A. V. Roiter. Representations of finite-dimensional algebras. With a chapter by B. Keller. Encyclopaedia Math. Sci., 73, Algebra, VIII, 1-177, Springer, Berlin, 1992.

[15] D. Herbera and J. Trlifaj. Almost free modules and Mittag-Leffler conditions. Adv. Math. 229 (2012), no. 6, 3436-3467.

[16] H. L. Hiller and S. Shelah. Singular cohomology in L. Israel J. Math. 26 (1977), no. 3-4, 313-319.

[17] K. Kunen. Set theory. Studies in Logic (London), 34. College Publications, London, 2011.

[18] D. Martin and R. Solovay. Internal Cohen extensions. Ann. Math. Logic 21970 no. 2, 143-178.

[19] H. Minamoto. Ampleness of two-sided tilting complexes. Int. Math. Res. Not. IMRN 2012, no. $1,67-101$.

[20] S. Shelah. Infinite abelian groups, Whitehead problem and some constructions. Israel J. Math. 18 (1974), 243-256.

[21] R. Solovay and S. Tennenbaum. Iterated Cohen extensions and Souslin's problem. Ann. of Math. (2) 94 (1971), 201-245.

[22] J. Trlifaj. Non-perfect rings and a theorem of Eklof and Shelah. Comment. Math. Univ. Carolin. 32 (1991), no. 1, 27-32.

Department of Mathematics, Tokyo University of Science, 1-3 Kagurazaka, SHINJUKU-KU, TOKYO 162-8601, JAPAN.

E-mail address: itaba@rs.tus.ac.jp

Faculty of Science, Shizuoka University, Ohya 836, Shizuoka, 422-8529, JAPAN.

E-mail address: diego.mejia@shizuoka.ac.jp

Faculty of Science, Shizuoka University, Ohya 836, Shizuoka, 422-8529, JAPAN.

E-mail address: yorioka@shizuoka.ac.jp 